\title{
Besonderheiten im pulmonalen NO-Stoffwechsel bei Patienten mit zystischer Fibrose
}

\section{H. Grasemann \\ F. Ratjen}

\author{
Pulmonary Metabolism of Nitric Oxide (NO) in Patients with Cystic Fibrosis
}

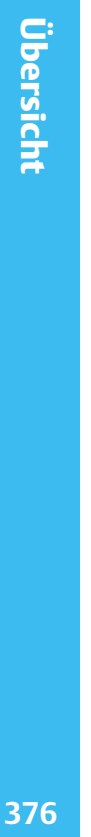

\section{Zusammenfassung}

In den Atemwegen generiertes Stickstoffmonoxid (NO) und seine Metabolite sind an einer Vielzahl physiologischer und pathophysiologischer Prozesse beteiligt. Unter anderem relaxiert NO die bronchiale Muskulatur, verbessert die Motilität der Zilien, hat antimikrobielle Effekte, und erhöht die Expression des CFTR(cystic fibrosis transmembrane regulation)Proteins in Atemwegsepithelzellen. Interessanterweise finden sich in den Atemwegen von Patienten mit zystischer Fibrose (CF) erniedrigte Konzentrationen von NO und von bioaktiven S-Nitrosothiolen (SNOs). Im Vergleich zu Patienten mit einer relativ normalen pulmonalen NO-Synthese weisen CF-Patienten mit niedrigen NO-Konzentrationen eine signifikant schlechtere Lungenfunktion auf und neigen vermehrt zu einer Besiedlung der Atemwege mit pathogenen Keimen wie P. aeruginosa. Als Konsequenz aus diesen Beobachtungen werden nun erste klinische Studien durchgeführt, die möglichen Effekte einer Erhöhung der bronchialen NO-Konzentration bei CF-Patienten untersuchen.

\section{Abstract}

Airway nitric oxide (NO) and its metabolites are involved in a number of physiological and pathophysiological processes. For instance, NO relaxes airway smooth muscle, improves airway ciliary motility, has antimicrobial effects, and increases expression of the CFTR (cystic fibrosis transmembrane regulator) protein in airway epithelial cells. Of interest, concentrations of NO and of bioactive S-nitrosothiols (SNOs) are decreased in the airways of patients with cystic fibrosis (CF). When compared to patients with relatively normal pulmonary NO formation, CF patients with low NO-concentrations have a significantly reduced pulmonary function and a higher frequency of bacterial colonisation of the airways with pathogens such as P. aeruginosa. As a consequence of these observations clinical trails have now been initiated to study possible effects of an augmented bronchial NO-concentration in CF-patients.

\section{Einleitung}

Das reaktive Sauerstoffradikal Stickstoffmonoxid (NO) ist als endogener Botenstoff in den Atemwegen an einer Vielzahl biologischer Prozesse beteiligt, zu denen die Regulation von Gefäßmuskeltonus und vaskulärer Permeabilität, die Neurotransmission, sowie die Modulation von Immunfuktion und Infektabwehr gezählt werden. NO wird enzymatisch durch sogenannte NO-Synthasen (NOS) produziert. Man unterscheidet die neuronale (NOS1) und die endotheliale NOS (NOS3), die konstitutiv exprimiert werden und deren NO-Synthese überwiegend von der zy- tosolischen $\mathrm{Ca}^{2+}$-Konzentration abhängt, von der induzierbaren Isoform (NOS2), deren Aktivität auf transkriptioneller Ebene geregelt wird. Alle drei NO-Synthasen werden in der Lunge von einer Vielzahl unterschiedlicher Gewebe exprimiert, unter anderem von Nerven-, Mukel- und Epithelzellen.

In den Atemwegen produziertes NO tritt in die Gasphase über und lässt sich daher in der Atemluft messen. Die Fraktion des exhalierten $\mathrm{NO}\left(\mathrm{FE}_{\mathrm{NO}}\right)$ ist bei Patienten mit entzündlichen Erkrankungen der Atemwege, wie dem Asthma bronchiale oder bei Allergien, erhöht. Da sich die $\mathrm{FE}_{\mathrm{NO}}$ bei Asthmatikern unter einer 
anti-inflammatorischen Therapie mit z.B. Kortikosteroiden verringert, hat sich die NO-Messung als nicht-invasive Methode zur Erfassung und Kontrolle einer Inflammation der Atemwege bei Asthmatikern etabliert. Die biologische Halbwertszeit von NO ist gering, da es schnell zu einer Vielzahl von Metaboliten reagiert. Diese NO-Metabolite haben in den Atemwegen zahlreiche, teils entgegengesetzte Wirkungen. Zum Beispiel kann NO durch die Bildung von Nitrosothiolen (SNOs) bronchodilatorisch wirken, oder kann zu pro-inflammatorischen Molekülen wie Peroxynitrit $\left(\mathrm{OONO}^{-}\right)$metabolisiert werden [1].

Der zystischen Fibrose (CF) liegt ein hereditärer Defekt eines Chloridionenkanals (CFTR; cystic fibrosis transmembrane conductance regulator) zugrunde. Bei der pulmonalen Manifestation der $\mathrm{CF}$, die gegenwärtig für nahezu $90 \%$ der krankheitsspezifischen Mortalität verantwortlich ist, sind entzündliche Prozesse und die Kolonisation der Atemwege mit pathogenen Keimen von maßgeblicher Bedeutung [2]. In dieser Übersichtsarbeit werden spezifische Besonderheiten in der Synthese und dem Metabolismus von pulmonalem NO bei der CF zusammenfassend dargestellt.

$\mathrm{FE}_{\mathrm{NO}}$ und NO-Metabolite als Marker der Inflammation in den CF-Atemwegen

Die $\mathrm{FE}_{\mathrm{No}}$ ist bei inflammatorischen Erkrankungen der Atemwege, wie akuten viralen Infekten, Bronchiektasie oder dem Asthma bronchiale erhöht. Beim Asthma ist dies vermutlich Folge einer vermehrten Expression von NOS2 in den Epithelzellen der Atemwege. An kultivierten Zellen lässt sich die zytokininduzierte Expression von NOS2 mit Steroiden wirkungsvoll unterdrücken. Ähnlich kommt es bei Asthmatikern unter einer antiinflammatorischen Therapie, mit z. B. Kortikosteroiden oder Leukotrienantagonisten, zu einem Absinken der $\mathrm{FE}_{\mathrm{No}}[3,4]$. Da die Messung von NO in der Atemluft nicht invasiv ist, wird sie für die Verlaufskontrolle der Entzündungsaktivität bei Asthmatikern mittlerweile von zahlreichen Zentren empfohlen.

Bei Patienten mit CF kommt es auf dem Boden einer Dehydratation der endobronchialen Sekrete zu einer Behinderung der mukoziliären Clearance [5]. Auf dem Boden dieser Veränderungen kommt es früh zu einer bakteriellen Besiedlung der Atemwege mit pathogenen Keimen wie Staph. aureus und P. aeruginosa. Zusätzlich zeigt sich das Bild einer ausgeprägten bronchialen Inflammation. Die chronische Zerstörung anatomischer Strukturen und der Umbau der Lunge mit Bildung von Bronchiektasen und Fibrose führt letztlich zur respiratorischen Insuffizienz. Mit der frühzeitigen antibiotischen Therapie einer Atemwegsinfektion versucht man den Prozess der Inflammation zu verlangsamen [6]. Verlässliche und sensible Marker, mit denen die Inflammation in den Atemwegen von CF-Patienten nicht-invasiv gemessen werden kann, stehen derzeit jedoch nicht zur Verfügung. Daher wurde von uns und anderen der Wert der NO-Messung bei Patienten mit CF untersucht. In diesen Studien fand sich überraschend, dass die $\mathrm{FE}_{\mathrm{NO}}$ bei CF-Patienten sowohl in den oberen [7] als auch in den unteren Atemwegen gegenüber Lungengesunden signifikant vermindert ist $[8,9]$. In einer Untersuchung an 30 Lungengesunden und 27 klinisch stabilen CF-Patienten mit einer FVC von $72 \pm 17 \%$ (Mittelwert \pm SD) und einer FEV $_{1}$ von $53 \pm 19 \%$ (Mittelwert \pm SD) des Sollwertes, lag die mittlere $( \pm S D) F_{N o}$ bei den Kontrollen bei 9,1 $\pm 3,6 \mathrm{ppb}$ (parts per billion) und bei den CF-Patienten bei $5,9 \pm 2,6 \mathrm{ppb}(\mathrm{p}<0,001$, t-Test) (Abb.1) [8]. Somit spiegelt die $\mathrm{FE}_{\mathrm{No}}$ bei $\mathrm{CF}$ nicht die Aktivität der Entzündung in den Atemwegen wider. Allerdings fanden sich in Untersuchungen unserer und anderer Arbeitsgruppen bei $\mathrm{CF}$ eine positive Korrelation zwischen $\mathrm{FE}_{\mathrm{NO}}$ und der Lungenfunktion, was vermuten lässt, dass bei CF-Patienten mit einer fortgeschrittenen Lungenerkrankung in den Atemwegen entweder weniger NO als bei Patienten mit guter Lungenfunktion generiert wird, oder dass Teile des produzierten NO in den Atemwegen retiniert werden $[8,10]$.

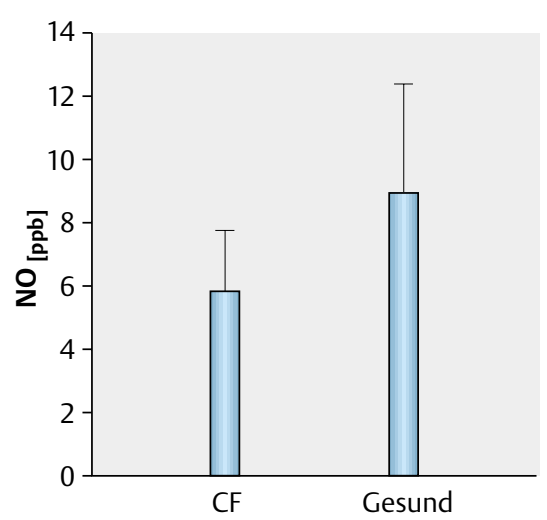

Abb. 1 Die Fraktion des exhalierten NO ( $\left.\mathrm{FE}_{\mathrm{NO}}\right)$ bei CF-Patienten und gesunden Kontrollen. Dargestellt sind Mittelwerte in ppb (parts per billion) und die einfache Standardabweichung. Die $\mathrm{FE}_{\mathrm{NO}}$ ist bei CF signifikant erniedrigt [8].

Die biologische Halbwertszeit von NO beträgt nur wenige Sekunden. Als reaktives Sauerstoffradikal wird NO rasch zu einer Vielzahl von Stickoxiden (NOx) mit unterschiedlicher Lebensdauer verwandelt, von denen Nitrit $\left(\mathrm{NO}_{2}\right)$ und Nitrat $\left(\mathrm{NO}_{3}\right)$ als stabile Endprodukte gelten [1]. Das in den Flüssigkeiten der Atemwege gelöste Nitrit und Nitrat reflektiert daher die pulmonale NO-Synthese. Dies konnte in einer Studie belegt werden, in der der BAL/ Serum-Quotient für Nitrat bei Lungengesunden etwa 15-mal höher lag als für Harnstoff [11]. In der gleichen Studie waren die Konzentrationen von Nitrat in der BAL-Flüssigkeit von Kindern mit Pneumonie gegenüber Gesunden erhöht, was vermutlich eine gesteigerte NO-Synthese bei den Kindern mit Pneumonie reflektiert [11]. Das CF-Bronchialepithel ist mit viskösem Schleim bedeckt. Dieser Schleim könnte als eine Diffusionsbarriere für das in den Atemwegen gebildete NO wirken. Die bei CF beobachtete erniedrigte $\mathrm{FE}_{\mathrm{NO}}$ könnte daher, neben einer verminderten Produktion, auch eine vermehrte Retention von NO in diesen Sekreten widerspiegeln. Tatsächlich fanden sich im Endotrachealsekret lungengesunder Kontrollen signifikant niedrigere Konzentrationen der stabilen NO-Metabolite Nitrat und Nitrit als im Sputum klinisch stabiler CF-Patienten (Abb. 2) [12]. Patienten mit einer pulmonalen Exazerbation der $\mathrm{CF}$ unterschieden sich dabei nicht von klinisch stabilen Patienten. Allerdings kam es unter einer 14-tägigen intravenösen antibiotischen Therapie zu einem signifikanten Anstieg der Nitratkonzentrationen im Sputum. Dieser Anstieg der Nitratkonzentration beruht vermutlich auf einer Reduktion der NOx-metabolisierenden Keime (z.B. P. aeruginosa), von denen das Bronchialsystem der CF-Patienten besiedelt wird. Zusätzlich fand sich in dieser Studie, ähnlich wie 


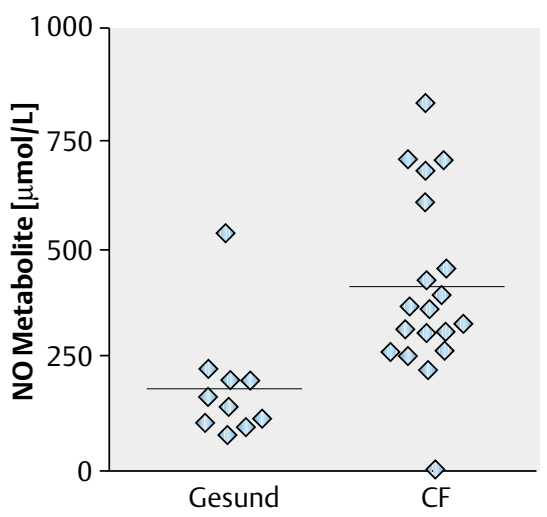

Abb. 2 Konzentrationen der stabilen NO-Metabolite (Nitrat plus Nitrit) im Endotrachealsekret Lungengesunder und im Sputum von CFPatienten. Die horizontale Linie stellt den jeweiligen Mittelwert dar. Die Konzentration der NO-Metabolite liegt bei CF signifikant höher als bei den Kontrollen [12].

schon zuvor für $\mathrm{FE}_{\mathrm{NO}}$, eine positive Korrelation zwischen $\mathrm{NOx}-$ Konzentration im Sputum und Lungenfunktion. Erhöhte NOxKonzentrationen im Sputum von $\mathrm{CF}$ mit akuter Exazerbation wurden auch von Linnane et al. beschrieben, allerdings wurden in dieser Studie keine Unterschiede zwischen stabilen CF-Patienten und den Kontrollpersonen beobachtet [13]. Die Retention und Metabolisierung von NO in den Sekreten der CF-Atemwege beruht wahrscheinlich auf physikalischen Eigenschaften der Sekrete, da die Nitratkonzentrationen im Sputum positiv mit der Rigidität des Sputums korreliert [14]. Mit diesen Studien konnte also gezeigt werden, dass weder die $\mathrm{FE}_{\mathrm{NO}}$ noch die Konzentration der NO-Metabolite im Sputum zur Verlaufskontrolle der Inflammation in den Atemwegen von CF-Patienten herangezogen werden können.

\section{Die Rolle der neuronalen NO-Synthase (NOS1)}

Obwohl bekannt ist, dass alle drei NO-Synthasen (NOSs) in verschiedenen Zelltypen der Atemwege exprimiert werden, ist der genaue molekulare und zelluläre Ursprung des messbaren NO in den Atemwegen noch unklar. Bei einigen inflammatorischen Atemwegserkrankungen, wie dem Asthma, scheint der durch Zytokine induzierbaren NOS2 eine besondere Bedeutung zuzukommen. Bei CF jedoch ist die Expression von NOS2 in den Epithelzellen der Atemwege, trotz der chronisch fortschreitenden Inflammation, vermindert, was am Tiermodell und an kultivierten humanen Zellen gezeigt werden konnte und möglicherweise für die persistierende Atemwegsinflammation eine wichtige Bedeutung hat $[15,16]$. Dies trägt womöglich zu der niedrigen $\mathrm{FE}_{\mathrm{No}}$ der CF-Patienten bei, und lässt auch den Schluss zu, dass die $\mathrm{FE}_{\mathrm{NO}}$ bei CF - wenigstens teilweise - die Funktion der so genannten „konstitutiv“ exprimierten NOS widerspiegelt. Die Vorstellung, dass NOS1 zur $\mathrm{FE}_{\mathrm{NO}}$ in den Atemwegen beisteuert, wurde in Studien an Asthmatikern untermauert, in denen gezeigt werden konnte, dass natürlich vorkommende Varianten im Gen der NOS1 mit der Höhe der $\mathrm{FE}_{\mathrm{NO}}$ assoziiert sind [17,18].

Zur Untersuchung der Fragestellung, ob es bei CF ein Zusammenhang zwischen Varianten im Gen der NOS1 und der Höhe der $\mathrm{FE}_{\mathrm{No}}$ geben könnte, führten wir daher Genotypisierungen bei 75
Patienten mit CF durch, von denen 33 Frauen und 42 Männer waren. Das mittlere Alter der Patienten lag bei 14 Jahren (Bereich 5-40 Jahre), die mittlere FVC bei 78,0 $\pm 19,7 \%( \pm$ SD) und die $\mathrm{FEV}_{1}$ bei $68,4 \pm 24,8 \%$ des Sollwertes. Zum Zeitpunkt der Untersuchung befanden sich alle Patienten in klinisch stabilem Zustand und waren ohne Zeichen einer pulmonalen Exazerbation, ohne i.v. antibiotische Therapie und ohne systemische oder inhalative Steroide. Die Messung von $\mathrm{FE}_{\mathrm{NO}}$ und Lungenfunktion erfolgte während eines Routinebesuchs in der Ambulanz des Zentrums für Kinder- und Jugendmedizin des Universitätsklinikums oder der Abteilung für Pneumologie der Ruhrlandklinik Essen. Bei dieser Gelegenheit wurde auch Sputum für mikrobiologische Untersuchungen aserviert. Die Messung des end-exspiratorischen NO erfolgte bei einem konstanten exspiratorischen Fluss zwischen 100 und $200 \mathrm{ml} / \mathrm{s}$. Die Lungenfunktion wurde spirometrisch gemessen. Die Untersuchung der durch eine Blutentnahme gewonnenen DNA erfolgte mittels Polymerase Kettenreaktion (PCR) und der SSLP (simple sequence length polymorphism) Analyse. Die bei der PCR verwendeten Primer waren spezifisch für eine $\mathrm{AAT}_{(\mathrm{n})}$-Wiederholungssequenz in Intron 20 des NOS1-Gen. Mit dieser Methode wurden insgesamt acht unterschiedliche Allele identifiziert, die zwischen 9 und 16 AAT-Wiederholungen enthielten. Da die Größe der Allele einem bimodalen Verteilungsmuster unterlagen, mit Häufigskeitsgipfeln bei Allel 10 ( 10 Wiederholungen) (45,3\% aller Allele) sowie bei den Allelen 13 und 14 (41,3\% aller Allele), unterteilten wir die Population in zwei Gruppen. Die eine bestand aus Patienten, bei denen beide Allele mindestens 12 AAT enthielten und die andere Gruppe mit mindestens einem Allel kleiner als 12 AAT-Wiederholungen. Für $\mathrm{FE}_{\mathrm{NO}}$ fanden sich signifikante Unterschiede zwischen diesen Gruppen mit niedrigeren Werten in der Gruppe mit vielen AAT-Wiederholungen im Vergleich zu den Patienten mit wenigen AAT-Wiederholungen $(4,0 \pm 3,4$ vs. $6,4 \pm 6,5 \mathrm{ppb}$, $\mathrm{p}=0,027$, t-Test). Die Variabilität um den jeweiligen Mittelwert unterschied sich ebenfalls signifikant $(\mathrm{p}<0,003$, F-Test $)$ zwischen den Gruppen, mit einer höheren Variabilität in der Gruppe mit wenigen AAT-Repeats. Bei den CFTR $\Delta$ F508 homozygoten Patienten, die genetisch eine homogene Gruppe darstellen, war die inverse Relation zwischen $\mathrm{FE}_{\mathrm{NO}}$ und Länge der AAT-Wiederholungssequenz (Repeats) besonders ausgeprägt (2,3 $\pm 1,4$ vs. $5,3 \pm$ 3,9 ppb, p = 0,0006, t-Test) (Abb. 3). Darüber hinaus zeigte sich für die gesamte CF-Population eine signifikante Assoziation zwischen NOS1-Genotyp und Kolonisation der Atemwege mit P. aeruginosa. In der Gruppe der Patienten mit langen AAT-Wiederholungen (niedrige $\mathrm{FE}_{\mathrm{NO}}$ ) fanden sich signifikant mehr P. aeruginosa positive Patienten als bei den Trägern von NOS1-Genotypen, die mit einer hohen $\mathrm{FE}_{\mathrm{No}}$ assoziiert waren (65\% vs. $38 \%$ ). Ähnliche Unterschiede fanden sich auch in der Untergruppe der CFTR $\Delta$ F508 homozygoten Patienten für P. aeruginosa ( $71 \%$ vs. $31 \%)$ und A. fumigatus ( $43 \%$ vs. $10 \%$ ). Interessanterweise bestand die Assoziation zwischen NOS1-Genotyp und $\mathrm{FE}_{\mathrm{NO}}$ nicht nur in der gesamten CF-Population, sondern auch bei den Patienten, die zum Zeitpunkt der Untersuchung keine Besiedlung der Atemwege mit P. aeruginosa oder A. fumigatus zeigten [19]. Diese Beobachtung lässt daher vermuten, dass die niedrige $\mathrm{FE}_{\mathrm{NO}}$ der Patienten mit langen AAT-Wiederholungen in NOS1 nicht Folge, sondern Ursache einer Atemwegsbesiedlung durch diese Keime waren. Damit ergaben sich erstmals Hinweise, dass die NOS1 über einen NO-abhängigen Mechanismus an der Regulation der Infektabwehr in den CF-Atemwegen beteiligt ist.

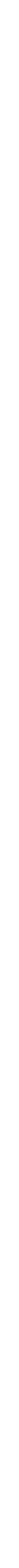




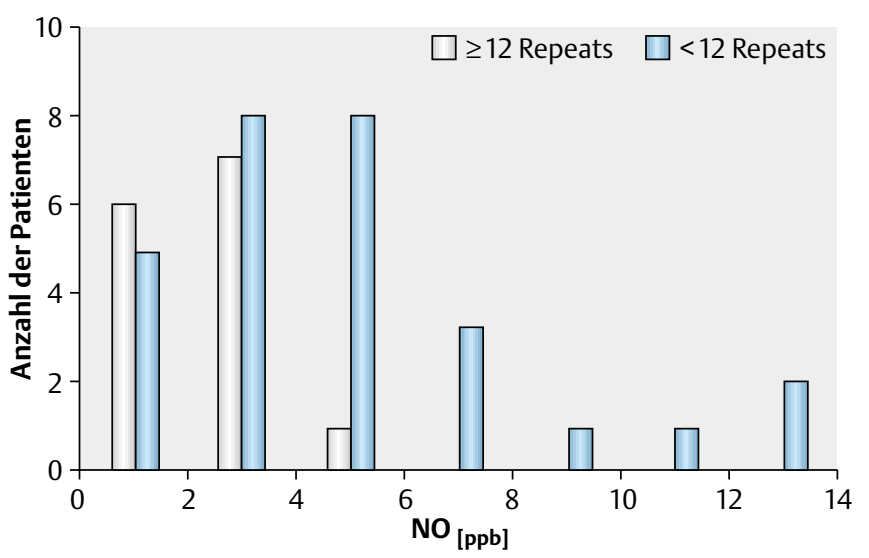

Abb. 3 Die $\mathrm{FE}_{\mathrm{NO}}$ bei CFTR $\Delta \mathrm{F} 508$ homozygoten CF-Patienten in Abhängigkeit vom Genotyp der neuronalen NO-Synthase (NOS1). Lange AAT-Wiederholungen ( $\geq 12$ Repeats) in Intron 20 sind mit einer niedrigen $\mathrm{FE}_{\mathrm{NO}}$ assoziiert [19].

Der überwiegende Teil des im Respirationstrakt generierten NO wird in den oberen Atemwegen gebildet, wobei die höchsten NOKonzentrationen in der Nase und den Nasennebenhöhlen gefunden werden. Da NO bakterio- und virostatisch wirkt, wird vermutet, dass in den oberen Atemwegen generiertes NO an der Abwehr von Infektionen des Respirationstraktes beteiligt ist. Interessanterweise erfolgt die Besiedlung der CF-Atemwege primär in den oberen Atemwegen. Dies konnte an Patienten gezeigt werden, die zwar einen positiven Rachenabstrich auf P. aeruginosa hatten, bei denen sich dieser Keim aber nicht in der BAL-Flüssigkeit fand [20]. Es ist daher vorstellbar, dass zwischen den reduzierten NO-Konzentrationen in den oberen Atemwegen von CFPatienten und der Kolonisation der Atemwege mit pathogenen Keimen ein Zusammenhang besteht. In der Nasenmukosa von Gesunden scheint NOS2 die überwiegend exprimierte Isoform der NO-produzierenden Enzyme darzustellen [21 - 23]. Da NOS2 an der Infektabwehr beteiligt ist, die Expression dieses Enzyms in den CF-Atemwegen aber signifikant vermindert ist $[15,16]$, könnte der NO-Synthese durch die konstitutiv exprimierte NOS1 eine besondere Bedeutung bei der CF zukommen. NOS1 konnte in der Nase mittels Immunohistochemie im Bereich von Gefäßen der Mukosa sowie in subepithelialen Drüsen und Nerven lokalisiert werden $[22,23]$. Daher untersuchten wir bei 40 klinisch stabilen CF-Patienten einen möglichen Zusammenhang zwischen NOS1-Genotyp, nasaler NO-Konzentration und Kolonisation der unteren Atemwege. Nasales NO wurde in der Luft gemessen, die den Patienten durch eine in einem Nasenloch platzierte Olive bei einem konstanten Sog (3L/Minute) aus der Nase gesaugt wurde. Zur Trennung der oberen von den unteren Atemwegen führten die Patienten während der NO-Messung ein Atemmanöver gegen einen positiven Atemwegswiderstand von $5 \mathrm{~cm}$ Wassersäule aus, der bei der Exhalation einen Verschluss des weichen Gaumensegels bewirkt. Die Zuteilung in die verschiedenen Gruppen erfolgte nach Größe der Allele in Intron 20 des NOS1-Gens, nach den gleichen Kriterien wie sie oben für die Assoziationsstudie zwischen NOS1 und $\mathrm{FE}_{\mathrm{NO}}$ beschrieben wurden. Bei dieser Untersuchung fand sich eine signifikante inverse Assoziation zwischen der Länge der Allele in NOS1 und den nasalen NO-Konzentrationen $(40,5 \pm 18,0$ vs. $72,6 \pm 38,9 \mathrm{ppb}, \mathrm{p}<$ 0,001, t-Test) (Abb. 4). Darüber hinaus lag der Anteil der P. aeruginosa positiven Patienten in der Gruppe der Patienten mit

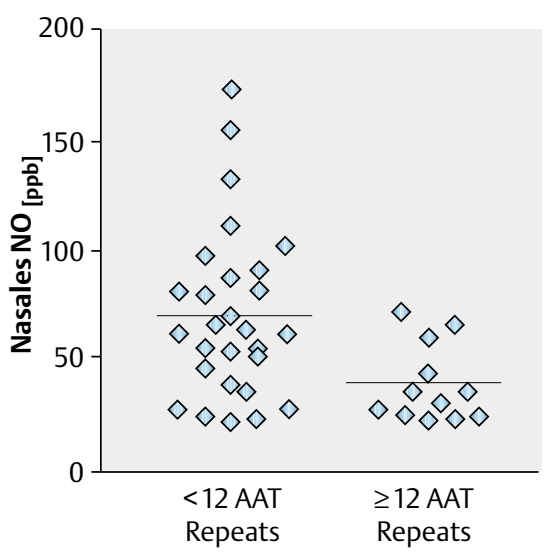

Abb. 4 Nasale NO-Konzentrationen bei CF-Patienten in Abhängigkeit vom NOS1-Genotyp. Die horizontalen Linien zeigen den jeweiligen Mittelwert [24].

NOS1-Genotypen, die mit niedrigem nasalen NO assoziiert waren, signifikant höher als bei Patienten mit hohen nasalen NOKonzentrationen ( $75 \%$ vs. $21 \%$ ) [24]. Aus dieser Studie lässt sich daher schließen, dass die CF-Patienten mit einer genetisch bedingt höheren NO-Synthese in den oberen Atemwegen einen besseren Schutz gegenüber Infekten der unteren Atemwege durch $P$. aeruginosa aufweisen.

\section{Therapeutische Ansätze zur Steigerung der bronchialen NO-Konzentration bei CF}

In den Atemwegen generiertes NO spielt eine wichtige Rolle bei der Regulation des bronchopulmonalen Widerstandes. Als Mediator des nicht-adrenergen nicht-cholinergen (NANC) Nervensystems vermittelt NO beim Menschen die induzierbare bronchiale Dilatation. Darüber hinaus besitzt NO eine bronchodilatative Wirkung, die über die Aktivierung von cAMP und Guanylatcyklase zu einer Relaxation glatter Muskulatur führt. Als Konsequenz aus den beschriebenen Beobachtungen einer erniedrigten $\mathrm{FE}_{\mathrm{NO}}$ in den CF-Atemwegen, sowie der Korrelation von niedriger $\mathrm{FE}_{\mathrm{NO}}$ mit schlechterer Lungenfunktion und erhöhter Anfälligkeit gegenüber einer Besiedlung des Bronchialsystems mit pathogenen Keimen, wurden nun erste klinische Studien initiiert, die mögliche Effekte einer Erhöhung der bronchialen NO-Konzentrationen bei CF-Patienten untersuchten.

In einer Studie, an der 13 CF-Patienten zwischen 14 und 38 Jahren sowie neun gesunde Probanden teilnahmen, wurde der Effekt von exogen zugeführtem NO auf die Lungenfunktion untersucht. Die Inhalation von NO-haltigem Gas in Konzentrationen von 100 und 1000 parts per billion (ppb), sowie 40 parts per million (ppm) über jeweils fünf Minuten führte zu keiner messbaren Veränderung von spirometrischen Daten oder der transkutanen Sauerstoffsättigung. Allerdings kam es bei acht CF-Patienten unter der Inhalation von 40 ppm NO zu einem leichten, wenn auch statistisch nicht signifikanten Anstieg der Nitratspiegel im Sputum (399 \pm 231 vs. $556 \pm 474 \mu \mathrm{mol} / \mathrm{L})$ [25]. Demnach hat die kurzfristige Inhalation von NO in Konzentrationen, wie sie physiologisch in den oberen Atemwegen Lungengesunder vorkommen $(100 \mathrm{ppb})$ und in einer Konzentration, die in der vasodilata- 
tiven Therapie der pulmonalen Hypertonie verwendet wird (40 ppm), bei CF-Patienten keinen unmittelbaren Effekt auf Parameter der Lungenfunktion.

In einer weiteren Studie wurde bei 11 CF-Patienten und 11 gesunden Kontrollen der Effekt einer Infusion von L-Arginin ( $500 \mathrm{mg} / \mathrm{kg} \mathrm{KG),} \mathrm{dem} \mathrm{Substrat} \mathrm{der} \mathrm{NO-Synthasen} \mathrm{(NOS),} \mathrm{auf} \mathrm{die}$ nasalen und exhalierten NO-Konzentrationen untersucht. Bei den gesunden Probanden erfolgte der maximale Anstieg der NOKonzentrationen in der Nase (180\%) und in den unteren Atemwegen (140\%) jeweils unmittelbar nach der Gabe von L-Arginin. Dieser Effekt war auch noch bei der Messung nach drei Stunden nachweisbar. Bei den CF-Patienten kam es ebenfalls unmittelbar nach der L-Arginin-Infusion zu einem signifikanten Anstieg der NO-Konzentrationen in den oberen $(190 \%)$ und den unteren Atemwegen (160\%), der auch noch nach drei Stunden nachweisbar war. Bei den Messungen nach 24 Stunden unterschieden sich die Mittelwerte in den Gruppen nicht mehr signifikant von den jeweiligen Ausgangswerten [26]. Somit konnte gezeigt werden, dass der verringerten $\mathrm{FE}_{\mathrm{NO}}$ bei $\mathrm{CF}$ kein Substratmangel von L-Arginin zugrunde liegt. Allerdings konnte trotz der Erhöhung der $\mathrm{FE}_{\mathrm{NO}}$ kein Effekt von L-Arginin auf die Lungenfunktion der CF-Patienten erreicht werden. Dies könnte daran gelegen haben, dass es, trotz der signifikanten Erhöhung der NO-Synthese durch die intravenöse Gabe von L-Arginin, bei den CF-Patienten zu keiner Normalisierung der NO-Konzentrationen in den Atemwegen kam. In der gleichen Studie wurde zusätzlich der Effekt einer L-Arginin-Infusion bei Patienten mit primärer ziliarer Dyskinesie untersucht. Bei diesen Patienten, die ebenfalls über eine signifikant verminderte endogene NO-Synthese verfügen, führte die Infusion von $\mathrm{NO}$, ähnlich wie bei $\mathrm{CF}$, auch zu einem vorübergehenden Anstieg der $\mathrm{FE}_{\mathrm{NO}}$ [26].

In einer erst kürzlich publizierten klinischen Studie wurde bei CF-Patienten der Effekt einer Inhalation von S-Nitrosoglutathione (SNO) untersucht [27]. Bei SNO handelt es sich um einen biologisch aktiven NO-Metaboliten, der in den Atemwegen bronchodilatatorische und antimikrobielle Aktivitäten besitzen. Die Konzentrationen von SNO sind in den Atemwegen von CF-Patienten erniedrigt [28]. Ein Ersatz dieses Metaboliten erscheint daher sinnvoll. Tatsächlich konnten Snyder et al. in ihrer doppelblinden, plazebokontrollierten Studie zeigen, dass die einmalige Inhalation von $0,05 \mathrm{ml} / \mathrm{kg}$ einer $10 \mathrm{mM}$ SNO-haltigen Lösung zu einem Anstieg von $\mathrm{FE}_{\mathrm{No}}$ und Sauerstoffsättigung führten [27]. Ein Effekt auf die Lungenfunktion konnte nach dieser einmaligen Gabe von SNO nicht beobachtet werden. Allerdings ist kürzlich in vitro ein Effekt von GSNO auf die CFTR-Expression von Epithelzellen gezeigt worden [29]. Die Ergebnisse dieser Studie legen nahe, dass eine NO-Ersatztherapie bei CF-Patienten möglich und sinnvoll sein könnte.

\section{Schlussfolgerungen}

Der NO-Stoffwechsel in den Atemwegen von Patienten mit CF unterliegt krankheitsspezifischen Besonderheiten, die in der Pathophysiologie der pulmonalen Erkrankung eine besondere Rolle zu spielen scheinen. Die Mehrzahl der Untersuchungen spricht dafür, dass $\mathrm{CF}$ mit einer verminderten NO-Produktion in den Atemwegen einhergeht. Da NO eine wichtige Rolle in vielen für die Erkrankung relevanten Funktionen, wie Stimulation der Zi- lienfunktion, Regulation des Bronchialmuskeltonus, und Abwehr von Bakterien wie z.B. P. aeruginosa besitzt, könnte sich eine Augmentation der NO-Produktion in den Atemwegen von CF-Patienten positiv auf den Krankheitsverlauf auswirken. In ersten klinischen Studien wird daher nun der Effekt einer pharmakologischen Manipulation der endogenen NO-Formation untersucht.

\section{Literatur}

${ }^{1}$ Gaston B, Drazen JM, Loscalzo J et al. The biology of nitrogen oxides in the airways. Am J Respir Crit Care Med 1994; 149: 538 - 551

2 Paul K, Ratjen F, Schuster A. Pulmonale Manifestation der Cystischen Fibrose. Monatsschr Kinderh 2001; 149: 222 - 238

${ }^{3}$ Massaro AF, Gaston B, Kita D et al. Expired nitric oxide levels during treatment of acute asthma. Am J Respir Crit Care Med 1995; 152: $800-803$

${ }^{4}$ Bisgaard $\mathrm{H}$, Loland L, Oj JA. NO in exhaled air of asthmatic children is reduced by the leukotriene receptor antagonist montelukast. Am J Respir Crit Care Med 1999; 160: 1227-1231

${ }^{5}$ Worlitzsch D, Tarran R, Ulrich M et al. Effects of reduced mucus oxygen concentration in airway Pseudomonas infections of cystic fibrosis patients. J Clin Invest 2002; 109: 317 - 325

${ }^{6}$ Ratjen F. Changes in strategies for optimal antibacterial therapy in cystic fibrosis. Int J Antimicrob Agents 2001; 17: 93 -96

${ }^{7}$ Balfour-Lynn IM, Laverty A, Dinwiddie R. Reduced upper airway nitric oxide in cystic fibrosis. Arch Dis Child 1996; 75: 319-322

${ }^{8}$ Grasemann H, Michler E, Wallot M et al. Decreased concentration of exhaled nitric oxide (NO) in patients with cystic fibrosis. Pediatr Pulmonol 1997; 24: 173-177

${ }^{9}$ Grasemann H, Ratjen F. Cystic fibrosis lung disease: the role of nitric oxide. Pediatr Pulmonol 1999; 28: $442-448$

${ }^{10} \mathrm{Ho}$ LP, Innes JA, Greening AP. Exhaled nitric oxide is not elevated in the inflammatory airways diseases of cystic fibrosis and bronchiectasis. Eur Respir J 1998; 12: 1290 - 1294

${ }^{11}$ Grasemann H, Ioannidis I, de Groot H et al. Metabolites of nitric oxide in the lower respiratory tract of children. Eur J Pediatr 1997; 156: $575-578$

${ }^{12}$ Grasemann H, Ioannidis I, Tomkiewicz RP et al. Nitric oxide metabolites in cystic fibrosis lung disease. Arch Dis Child 1998; 78: 49-53

${ }^{13}$ Linnane SJ, Keatings VM, Costello CM et al. Total sputum nitrate plus nitrite is raised during acute pulmonary infection in cystic fibrosis. Am J Respir Crit Care Med 1998; 158: 207-212

${ }^{14}$ Grasemann H, Tomkiewicz RP, Ioannidis I et al. Metabolites of nitric oxide and viscoelastic properties of airway secretions in cystic fibrosis. Am J Respir Crit Care Med 1997; 155: A46

${ }^{15}$ Kelley TJ, Drumm ML. Inducible nitric oxide synthase expression is reduced in cystic fibrosis murine and human airway epithelial cells. J Clin Invest 1998; 102: 1200-1207

${ }^{16}$ Meng QH, Springall DR, Bishop AE et al. Lack of inducible nitric oxide synthase in bronchial epithelium: a possible mechanism of susceptibility to infection in cystic fibrosis. J Pathol 1998; 184: 323-331

${ }^{17}$ Wechsler ME, Grasemann H, Deykin A et al. Exhaled nitric oxide in patients with asthma. Association with NOS1 genotype. Am J Respir Crit Care Med 2000; 162: $2043-2047$

18 Grasemann H. Die Genetik der neuronalen NO-Synthase (NOS1) in der Ätiologie des Asthma bronchiale. Pneumologie 2001; 55: 390 - 395

${ }^{19}$ Grasemann H, Knauer N, Büscher R et al. Airway nitric oxide levels in cystic fibrosis patients are related to a polymorphism and the neuronal nitric oxide synthase gene. Am J Respir Crit Care Med 2000; 162: $2172-2176$

${ }^{20}$ Rosenfeld M, Emerson J, Accurso F et al. Diagnostic accuracy of oropharyngeal cultures in infants and young children with cystic fibrosis. Pediatr Pulmonol 1999; 28: 321 - 328

21 Watkins DN, Peroni DJ, Basclain KA et al. Expression and activity of nitric oxide synthase in human airway epithelium. Am J Respir Cell Mol Biol 1997; 16: 629-539

${ }^{22}$ Tasman AJ, Bogatzki B, Heppt W et al. Nitric oxide synthase in the innervation of the human nasal mucosa: correlation with neuropeptides and thyrosine hydroxylase. Laryngoscope 1998; 108: 128-133

${ }^{23}$ Riederer A, Held B, Mayer B et al. Histochemical and immunocytochemical study of nitrergic innervation in human nasal mucosa. Ann Otol Rhinol Laryngol 1999; 108: 869-875 
${ }^{24}$ Grasemann H, Storm van's Gravesande K et al. Nasal nitric oxide levels are associated with a polymorphism in the neuronal nitric oxide synthase (NOS1) genes in cystic fibrosis patients. Nitric Oxide 2002; 6 : 236-241

${ }^{25}$ Ratjen F, Kavuk I, Gärtig S et al. Airway nitric oxide in infants with acute wheezy bronchitis. Pediatr Allergy Immunol 2000; 11: 230-235

${ }^{26}$ Grasemann H, Gärtig SS, Wiesemann HG et al. Effect of L-arginine infusion on airway NO in cystic fibrosis and primary ciliary dyskinesia syndrome. Eur Respir J 1999; 13: 114 - 118

27 Snyder AH, McPherson ME, Hunt JF et al. Acute effects of aerosolized S-nitrosoglutathione in cystic fibrosis. Am J Respir Crit Care Med 2002; 165: $922-926$

28 Grasemann H, Gaston B, Fang K et al. Decreased levels of nitrosothiols in the lower airways of patients with cystic fibrosis and normal pulmonary function. J Pediatr 1999; 135: 770 - 772

${ }^{29}$ Zaman K, McPherson M, Vaughan J et al. S-nitrosoglutathione increases cystic fibrosis transmembrane regulator maturation. Biochem Biophys Res Commun 2001; 284: 65-70

\section{Information}

\section{Neuer Vorstand der Mitteldeutschen Gesellschaft für Pneumologie (MDGP)}

Am 4. 5. 2002 fanden in Weimar Legefeld die Mitgliederversammlung der MDGP und die Vorstandswahlen statt.

Zum neuen Präsidenten der Mitteldeutschen Gesellschaft für Pneumologie wurde Prof. Dr. med. Dr. rer. nat. Claus Kroegel, Friedrich-Schiller-Universität Jena, gewählt.

Weiter im neuen Vorstand vertreten sind:

Prof. Dr. G. Liebetrau, Lungenklinik Lostau

(Stellvertretender Präsident)

DM U. Heschel, Greiz (Schriftführer)

Priv.-Doz. Dr. K. Harzbecker, Aue (Schatzmeister)

MR Dr. M. Franke, Sonneberg (Geschäftsführer)

Dr. E. Laake, Dresden

Dr. K.-T. Schulz, Magdeburg

Wir wünschen dem neu gewählten Vorstand viel Glück und Erfolg bei seiner Arbeit zum Wohle und zur Förderung der Pneumologie, insbesondere in den mitteldeutschen Ländern.

Die Förderung des pneumologischen Nachwuchses steht im Mittelpunkt der zukünftigen Aufgaben. Nicht zuletzt deshalb hat die MDGP in diesem Jahr erneut den von der Fukisawa Deutschland $\mathrm{GmbH}$ gestifteten und mit $€ \mathbf{5 0 0 0}$,- dotierten Förderpreis für junge Nachwuchswissenschaftler ausgeschrieben. 